\title{
Common variants near CAV1 and CAV2 are associated with primary open-angle glaucoma
}

\author{
Gudmar Thorleifsson ${ }^{1}$, G Bragi Walters ${ }^{1}$, Alex W Hewitt ${ }^{2,3}$, Gisli Masson ${ }^{1}$, Agnar \\ Helgason $^{1,4}$, Andrew DeWan ${ }^{5}$, Asgeir Sigurdsson ${ }^{1}$, Adalbjorg Jonasdottir ${ }^{1}$, Sigurjon A \\ Gudjonsson ${ }^{1}$, Kristinn P Magnusson ${ }^{6}$, Hreinn Stefansson ${ }^{1}$, Dennis S C Lam ${ }^{7}$, Pancy O S \\ Tam $^{7}$, Gudrun J Gudmundsdottir 8,9 , Laura Southgate ${ }^{10}$, Kathryn P Burdon ${ }^{3}$, Maria Soffia \\ Gottfredsdottir $^{11}$, Micheala A Aldred ${ }^{12}$, Paul Mitchell ${ }^{13}$, David St Clair ${ }^{14}$, David A \\ Collier ${ }^{15,16}$, Nelson Tang ${ }^{17}$, Orn Sveinsson ${ }^{18}$, Stuart Macgregor ${ }^{19}$, Nicholas G Martin ${ }^{19}$, \\ Angela J Cree ${ }^{20}$, Jane Gibson ${ }^{21}$, Alex MacLeod ${ }^{22}$, Aby Jacob ${ }^{22}$, Sarah Ennis ${ }^{21}$, Terri L \\ Young ${ }^{23}$, Juliana C N Chan ${ }^{24}$, Wojciech S S Karwatowski ${ }^{25}$, Christopher J Hammond ${ }^{26}$, \\ Kristjan Thordarson ${ }^{27}$, Mingzhi Zhang ${ }^{28}$, Claes Wadelius ${ }^{29}$, Andrew J Lotery ${ }^{20,22}$, Richard \\ C Trembath ${ }^{10}$, Chi Pui Pang ${ }^{7}$, Josephine Hoh ${ }^{5}$, Jamie E Craig ${ }^{3}$, Augustine Kong ${ }^{1}$, David A \\ Mackey $^{2,30,31}$, Fridbert Jonasson ${ }^{11,32}$, Unnur Thorsteinsdottir ${ }^{1,32}$, and Kari Stefansson ${ }^{1,32}$
}

${ }^{1}$ deCODE genetics Inc, Reykjavik, Iceland ${ }^{2}$ Centre for Eye Research Australia, University of Melbourne, Royal Victorian Eye and Ear Hospital, Melbourne, Australia ${ }^{3}$ Department of Ophthalmology, Flinders University, Flinders Medical Centre, Adelaide, Australia ${ }^{4}$ University of Iceland, Reykjavik, Iceland ${ }^{5}$ Department of Epidemiology and Public Health, Yale University, New Haven, Connecticut, USA ${ }^{6}$ Department of Biotechnology, University of Akureyri, Borgir vid Nordurslod, Akureyri, Iceland ${ }^{7}$ Department of Ophthalmology and Visual Sciences, The Chinese University of Hong Kong, Hong Kong, China ${ }^{8}$ Eye Clinic, Akranes, Iceland ${ }^{9}$ Eye Clinic Hamrahlid, Reykjavik, Iceland ${ }^{10}$ King's College London, Department of Medical and Molecular Genetics, School of Medicine, Guy's Hospital, London, UK ${ }^{11}$ Department of Ophthalmology National University Hospital, Reykjavik, Iceland ${ }^{12}$ Genomic Medicine Institute, Cleveland Clinic, Cleveland, Ohio, USA ${ }^{13}$ Centre for Vision Research, Department of Ophthalmology and Westmead Millennium Institute, University of Sydney, Westmead, Australia ${ }^{14}$ Department of Mental Health, University of Aberdeen, Royal Cornhill Hospital, Aberdeen, UK ${ }^{15}$ Division of Psychological Medicine, Institute of Psychiatry, King's College, London, UK ${ }^{16}$ Psychiatric Laboratory, Department of Psychiatry, West China Hospital, Sichuan University, Sichuan, China ${ }^{17}$ Laboratory for Genetics of Disease Susceptibility, Li Ka Shing Institute of Health Sciences, Faculty of Medicine, The Chinese University of Hong Kong, Hong Kong ${ }^{18}$ Eye Clinic, Mjodd, Reykjavik,

(C) 2010 Nature America, Inc. All rights reserved.

Correspondence should be addressed to G.T. (gudmar.thorleifsson@decode.is) or K.S. (kstefans@ decode.is).

Note: Supplementary information is available on the Nature Genetics website.

Author Contributions: The study was designed, the results interpreted and the first draft written by G.T., U.T., F.J. and K.S. The statistical analysis was performed by G.T., A.W.H. and A.K. A. Jonasdottir, A.S. and S.A.G. did the bioinformatic analysis of the $7 \mathrm{q} 31$ region, A.H. did the phylogenetic analysis and G.M. did the imputation. Genotyping at deCODE genetics was supervised by G.B.W. and U.T. Those responsible for case and control ascertainment, recruitment and phenotype information were F.J., G.J.G., H.S., K.P.M., M.S.G. and O.S. (Icelandic POAG cases and controls); K.P.M. and K.T. (Icelandic myopia cases); L.S., M.A.A., R.C.T. and W.S.S.K. (Leicester POAG cases); D.A.C. and D.St.C. (controls used for Leicester cases); A. Jacob, A.J.C., A.J.L., A.M. J.G. and S.E. (Southampton POAG cases and controls); C.W. (Swedish POAG cases and controls); P.M., C.J.H., N.G.M., S.M., T.L.Y., A.W.H., J.E.C., K.P.B. and D.A.M. (Australian POAG cases and controls and collection, genotyping and analysis of the Australian Twin study); C.P.P., D.S.C.L., P.O.S.T., A.D.W., J.H. and M.Z. (collection and genotyping of Hong Kong and Shantou POAG cases and unaffected controls); and J.C.N.C. and N.T. (population controls from Hong Kong). All authors contributed to the final version of the paper.

Competing Financial Interests: The authors declare competing financial interests: details accompany the full-text HTML version of the paper at http://www.nature.com/naturegenetics/. 
Iceland ${ }^{19}$ Genetics and Population Health, Queensland Institute of Medical Research, Brisbane, Australia ${ }^{20}$ Clinical Neurosciences Division, School of Medicine, University of Southampton, Southampton, UK ${ }^{21}$ Genetic Epidemiology and Bioinformatics Group, Human Genetics Division, School of Medicine, University of Southampton, Southampton, UK ${ }^{22}$ Southampton Eye Unit, Southampton University Hospital Trust, Southampton, UK ${ }^{23}$ Center for Human Genetics, Duke University Medical Center, Durham, North Carolina, USA ${ }^{24}$ Department of Medicine and Therapeutics, The Chinese University of Hong Kong, Prince of Wales Hospital, Shatin, New Territories, Hong Kong ${ }^{25}$ Department of Ophthalmology, University Hospitals of Leicester, Leicester, UK ${ }^{26}$ Department of Twin Research and Genetic Epidemiology, King's College London School of Medicine, St. Thomas' Hospital, London, UK ${ }^{27}$ Eye Clinic, Kringlan, Reykjavik, Iceland ${ }^{28}$ Joint Shantou International Eye Center, Shantou University and the Chinese University of Hong Kong, Shantou, China ${ }^{29}$ Department of Genetics and Pathology, Uppsala University, Uppsala, Sweden ${ }^{30}$ Lions Eye Institute, University of Western Australia, Centre for Ophthalmology and Visual Science, Perth, Australia ${ }^{31}$ Discipline of Medicine, Royal Hobart Hospital, University of Tasmania, Hobart, Australia ${ }^{32}$ Faculty of Medicine, University of Iceland, Reykjavik, Iceland

\section{Abstract}

We conducted a genome-wide association study for primary open-angle glaucoma (POAG) in 1,263 affected individuals (cases) and 34,877 controls from Iceland. We identified a common sequence variant at 7q31 (rs4236601[A], odds ratio $(\mathrm{OR})=1.36, P=5.0 \times 10^{-10}$ ). We then replicated the association in sample sets of 2,175 POAG cases and 2,064 controls from Sweden, the UK and Australia (combined OR $=1.18, P=0.0015$ ) and in 299 POAG cases and 580 unaffected controls from Hong Kong and Shantou, China (combined OR $=5.42, P=0.0021$ ). The risk variant identified here is located close to $C A V 1$ and $C A V 2$, both of which are expressed in the trabecular meshwork and retinal ganglion cells that are involved in the pathogenesis of POAG.

Glaucoma is the leading cause of irreversible blindness worldwide, affecting approximately 70 million people ${ }^{1}$. It is a chronic degenerative optic neuropathy with progressive loss of retinal ganglion cells and axons resulting in a corresponding thinning of the neuroretinal rim of the optic nerve and a characteristic visual field defect. It is distinct from other forms of optic neuropathy in that the neuroretinal rim of the optic nerve retains its normal pink color as it becomes progressively thinner, leading to an enlarged optic-nerve cup. POAG is the most common form of glaucoma. Excluding rare primary juvenile glaucoma with age of onset between 10 and 35 years, POAG is arbitrarily divided into high-pressure glaucoma (defined as $\geq 22 \mathrm{mmHg}$ ) and normal-pressure glaucoma. POAG is thought to have a multifactorial etiology, with the main risk factors being age, elevated intraocular (IOP) pressure, family history, race, central corneal thickness (CCT), hypertension, diabetes and myopia. The familiality of POAG has been known for decades, and studies have revealed three- to ninefold greater risk of POAG in first-degree relatives of POAG cases than in the population in general $^{2}$.

POAG is a genetically heterogeneous disease that shows linkage to at least 20 genetic loci ${ }^{3}$. Three genes predisposing to glaucoma have been isolated from these loci: MYOC (encoding myocilin) ${ }^{4}, O P T N$ (encoding optineurin) ${ }^{5}$ and WDR36 (encoding WD repeat domain 36$)^{6}$, although the association with WDR36 does not replicate in all populations. The variants in these genes are rare and may together contribute to 5-6\% of all POAG cases ${ }^{2}$. More recently, rare mutations in NTF4 have been found in individuals with $\mathrm{POAG}^{7}$, and a genome-wide association study (GWAS) yielded two common exonic variants in LOXL1 that explain over $99 \%$ of the cases with exfoliation glaucoma (XFG) in individuals of European ancestry ${ }^{8}$. This association with XFG has been replicated in several other 
populations of European, African and Asian ancestry, although the variants do not associate with POAG in these populations ${ }^{8,9}$. A recent GWAS conducted in a Japanese population identified three loci with suggestive evidence for association with $P O A G^{10}$, although this association was not replicated in an independent study in an Indian population ${ }^{11}$.

To search for genomic variants that confer risk of POAG, we conducted a GWAS on 1,263 POAG cases diagnosed by Icelandic ophthalmologists using established glaucoma criteria ${ }^{12}$ and 34,877 population controls from Iceland (Supplementary Note). After quality filtering, 303,117 SNPs typed with the Illumina HumanHap300 or HumanHapCNV370 BeadChips were tested for association with POAG. The results were adjusted for relatedness using the method of genomic controls ${ }^{13}$ by dividing the $\chi^{2}$ statistic by the genomic inflation factor 1.182 .

Two highly correlated SNPs, rs4236601[A] and rs1052990[G] ( $r^{2}=0.64$ based on the Utah (CEU) HapMap(r22) samples), reached genome-wide significance of $P<1.6 \times 10^{-7}$ (Supplementary Fig. 1 and Supplementary Table 1$)$. These variants, with $\mathrm{OR}=1.36(P=5.0$ $\left.\times 10^{-10}\right)$ and $\mathrm{OR}=1.32\left(P=1.1 \times 10^{-9}\right)$, respectively, are located within the same linkage disequilibrium (LD) block between $C A V 1$ and $C A V 2$ (encoding caveolin 1 and 2) on 7q31 (Table 1 and Fig. 1). After adjusting for the observed association with rs4236601[A], neither rs $1052990[\mathrm{G}]$ nor any other variant in the 7q31 region showed significant association with POAG (Supplementary Table 2). None of the variants described in a previous study ${ }^{10}$ or any other highly correlated variants associated with POAG in the Icelandic samples (Supplementary Table 3).

We typed rs4236601 in 200 POAG cases and 194 controls from Sweden, in 871 POAG cases and 865 controls from Leicester and Southampton, UK, and in 1,104 POAG cases and 1,001 controls from Australia. In the Swedish set, rs4236601[A] conferred similar risk of POAG as that observed in the Icelandic dataset $(\mathrm{OR}=1.33, P=0.092)$, whereas the estimated risk was less in the two UK sets $(\mathrm{OR}=1.14, P=0.2$ and $\mathrm{OR}=1.04, P=0.75)$ (Table 1). The Australian sample consisted of three studies—a study from Tasmania (GIST), a study from South Australia (ANZRAG) and the Blue Mountains Eye Study (BMES) - that individually have estimated $\mathrm{OR}=1.17(P=0.29), \mathrm{OR}=1.25(P=0.038)$ and $\mathrm{OR}=1.26(P$ $=0.13)$, respectively. Combined, the replication sets gave OR $=1.18(95 \%$ CI $1.06-1.31, P$ $=0.0015)$, and including the discovery set gave $\mathrm{OR}=1.27$ (95\% CI 1.18-1.36, $P=2.2 \times$ $\left.10^{-11}\right)$. There was heterogeneity in the effect estimates among the study populations $\left(P_{\text {het }}=\right.$ 0.048); in particular, the estimated effect in the samples from Southampton was low (Table 1). POAG is a heterogeneous disease and therefore this heterogeneity in the estimated effect sizes is not surprising. In the Southampton samples, the risk was confined to a subset of normal-pressure glaucoma cases, whereas we observed no risk for the majority of the cases diagnosed with high-pressure glaucoma (Supplementary Table 4). Higher risk in normalpressure cases was also observed, although not consistently, in the POAG cases from Iceland and Australia. rs4236601[A] did not associate with XFG in samples from Iceland and Sweden (Table 1).

The estimated population frequency of rs 4236601 [A] ranges from $20.7 \%$ to $28.1 \%$ in the four populations studied, and the corresponding population attributed risk percentage was $12 \%$, calculated using the mean of the population frequencies and the estimated OR of 1.27. About 6\% of the individuals in the four populations carry two copies of the risk allele, and their risk of developing POAG is 1.6 times greater than those carrying no risk variant.

The frequency of the risk variant rs4236601[A] differs between ethnicities. In the HapMap populations, the estimated frequency ranges from $45 \%$ in the Yoruba population and $28 \%$ in the Utah CEPH population to $2 \%$ in the Han Chinese population. We did not detect the 
variant in the 60 HapMap individuals from Japan. We tested the variant for association with POAG in 299 cases and 580 unaffected controls of Chinese origin from Hong Kong and Shantou (Table 1). Although the variant is rare, with a frequency of about $1.8 \%$ in cases and less than $0.4 \%$ in controls, the association was significant and yielded an OR of $5.42(P=$ 0.0021 ). We also tested the variant in 1,027 population controls from Hong Kong, where its frequency is slightly higher than in the unaffected control population and has a frequency of $0.7 \%$. The greater risk and lower frequency of rs4236601[A] in the Chinese population as compared to European populations raises the possibility that it tags some rare unknown causative variant through LD that is stronger in the Chinese population than in European populations. We note that in the Chinese (CHB) HapMap (r22) samples, 32 SNP alleles, spread across $174 \mathrm{~kb}$, are perfect surrogates $\left(r^{2}=1\right)$ of rs4236601, whereas in the Utah CEU HapMap sample, there are only five such SNP alleles covering $12.6 \mathrm{~kb}$ (Supplementary Fig. 2). Of the $32 \mathrm{CHB}$ surrogate SNPs, we tested 31 for association in the Icelandic sample set but observed no association independent of rs4236601 (Supplementary Table 2). Thus, either the risk attributable to this locus differs in European and Chinese populations or there remains an undetected rare causative variant that is not well tagged by existing SNPs in the Utah CEU HapMap samples.

To search for protein-coding mutations responsible for the association, we sequenced the promoter region, exons and exon-intron boundaries of $C A V 1$ and $C A V 2$ in 280 POAG cases and 358 controls from Iceland (Supplementary Note). SNPs identified through this effort were imputed into the remaining Icelandic POAG case and control samples using recently developed methods of long-range phasing of haplotypes in sets of related individuals ${ }^{14}$. Two of the identified SNPs, the nonsynonymous coding variant rs8940 and rs 1052990 located in the 3' untranslated region end of $C A V 2$, were also genotyped in the samples from Australia and Sweden. Although several of the identified variants showed significant association with POAG, none of the tested SNPs remained significant after adjusting for the effect of rs4236601[A] and none of them account for the association of rs4236601[A] with POAG (Supplementary Table 5 and Supplementary Note). This indicates that rs4236601 is unlikely to tag mutations within the coding region of $C A V 1$ or $C A V 2$.

To evaluate whether the $7 \mathrm{q} 31$ variant predisposes to POAG through known risk factors, we tested for association of rs4236601[A] with IOP, CCT, hypertension, type 2 diabetes (T2D) and myopia in 1,713 samples from the Twins Eye Study in Tasmania (TEST) ${ }^{15}$; in 691 Australian POAG cases and 439 controls with IOP measurements; in 316 samples with IOP and CCT measurements without glaucoma from the Reykjavik Eye Study; in 883 individuals from Iceland with spherical equivalent refraction error of -3 diopters or higher and in 2,251 T2D cases and in 34,647 controls and 7,007 hypertension cases and 31,521 controls from Iceland. Of the six traits tested, nominally significant association was only observed for increased IOP $(P=0.034$; Supplementary Table 6$)$.

The LD block containing rs4236601 contains two known genes, $C A V 1$ and $C A V 2$, and few uncharacterized expressed sequence tags. $C A V 1$ and $C A V 2$ are members of the caveolin gene family that also includes the muscle-specific $C A V 3$ gene. $C A V 1$ and $C A V 2$ are expressed in most human cell types, including tissues such as the scleral spur cells ${ }^{16}$, trabecular meshwork ${ }^{17}$ and retinal ganglion cells ${ }^{18}$ of the eye, but alterations in these tissues are thought to play a role in the pathology of POAG, leading to loss of retinal ganglion cell axons, along with supportive glia and vasculature. Notably, under experimental conditions, $C A V 1$ showed consistent upregulation in the trabecular meshwork after one hour of increased $\mathrm{IOP}^{19}$.

CAV1 and CAV2 are involved in the formation of caveolae which are specialized invaginations of the plasma membrane that are rich in cholesterol and other lipids, and they 
take part in transcytosis. However, it is the role of caveolae in signal transduction through interaction with signaling molecules that has been most extensively studied. Caveolae recruit and compartmentalize various signaling molecules through direct physical interaction mediated by the cave-olin scaffolding domain (CSD) in CAV1. This interaction generally results in inhibition of signaling ${ }^{20-23}$. Caveolins have been suggested as regulators of adult neural stem cell proliferation, as evidenced by increased proliferation of adult neural stem cells in Cav1, Cav2 and Cav3 knockout mice ${ }^{24}$. The regulation by CAV1 of the endothelial nitric oxide synthase (eNOS), an enzyme that produces nitric oxide, is well documented, but the interaction of CAV1 and eNOS leads to eNOS inactivation ${ }^{25,26}$ and reduced nitric oxide production. Nitric oxide plays an important role in the regulation of many physiological functions in the cardiovascular system and the central and peripheral nervous systems. Nitric oxide produced in excessive amounts causes cytotoxicity, neurodegeneration, apoptotic cell death and circulatory failure. In addition to nitric oxide signaling, CAV1 has been shown to be an important regulator of TGF- $\beta$ signaling through interaction with the TGF- $\beta$ type 1 receptor. Both nitric oxide and TGF- $\beta$ signaling have been implicated as culprits in the pathogenesis of $\mathrm{POAG}^{27,28}$.

We tested the effect of rs 4236601 on $C A V 1$ and $C A V 2$ mRNA expression measured in 747 blood samples and 606 adipose tissue samples ${ }^{29}$. No correlation between the POAG variant and $C A V 1$ or $C A V 2$ expression was observed (data not shown); however, as gene regulation can be highly tissue specific, the effect of rs4236601 on CAV1 and CAV2 expression in ocular tissue, which is more relevant for glaucoma than blood and adipose tissue and where the expression of $C A V 1$ or $C A V 2$ is more likely to influence or lead to disease, can not be excluded.

It is of interest to note that there was a recent report of an association of a SNP, rs3807989[A], within the same LD block as rs4236601 with the PR interval (an electrocardiogram measurement) and atrial fibrillation ${ }^{30}$. rs3807989 is weakly correlated with rs4236601 $\left(r^{2}<0.01\right)$ and does not associate with POAG, nor does rs4236601[A] associate with PR interval or atrial fibrillation. The CAVI-CAV2 locus thus adds to the growing list of loci where closely spaced signals show distinct associations with diverse traits.

We have identified a sequence variant, rs4236601[A], that is associated with POAG susceptibility in populations of European and east Asian ancestry. The variant does not have a major effect on known risk factors for POAG such as IOP and central corneal thickness, and it has not been associated with susceptibility to diseases such as T2D, hypertension or myopia that are all risk factors of POAG. This sequence variant is in the same LD block as $C A V 1$ and $C A V 2$. The frequency of the POAG variant differs between ethnicities; in particular, the frequency of the variant is much lower in east Asian populations than in individuals of European descent. These data highlight the importance of considering the genetic component in the risk of common complex diseases in the context of geographic ancestry.

\section{Methods}

Methods and any associated references are available in the online version of the paper at http://www.nature.com/naturegenetics/.

\section{Supplementary Material}

Refer to Web version on PubMed Central for supplementary material. 


\section{Acknowledgments}

We thank all the participants whose contribution made this study possible, as well as their ophthalmologists. We also thank the personnel at deCODE recruitment center and core facilities for their hard work and enthusiasm. We would also like to acknowledge A. Hill (University Hospitals of Leicester National Health Service (NHS) Trust) for invaluable help with sample collection and the Wellcome Trust for funding (programme grant 062346/Z/00/Z and project grant 078751/Z/05/Z). The authors acknowledge financial support from the UK Department of Health via the National Institute for Health Research (NIHR) comprehensive Biomedical Research Centre award to Guy's and St. Thomas' NHS Foundation Trust in partnership with King's College London and King's College Hospital NHS Foundation Trust. We also thank the following organizations for their financial support: Clifford Craig Medical Research Trust; Ophthalmic Research Institute of Australia; Pfizer Australia; Glaucoma Australia; American Health Assistance Foundation; the glaucoma research foundation and the Australian National Health and Medical Research Council (NHMRC); the International Glaucoma Association, UK and the Eire Glaucoma Society and Optegra UK Ltd. J.E.C. is supported in part by an NHMRC Practitioner Fellowship, and D.A.M. is a Pfizer Australia Research Fellow. We would also like to thank O. Wallerman, M. Jansson, L.-I. Larsson and L. Tomic for their assistance and the Swedish Research Council for financial support. The authors acknowledge the funding and support of the following organizations: the US National Institutes of Health (NIH)/National Eye Institute grant 1RO1EY018246 and the NIH Center for Inherited Diseases Research (CIDR) (PI: T. Young); the Verto Institute, the American Health Assistance Foundation (AHAF) National Glaucoma Research and the Ellison Foundation for Aging Research; and the Southampton Wellcome Trust Clinical Research Facility. We thank D.R. Nyholt, G. Montgomery, S. Medland, S. Gordon, A. Henders, B. McEvoy, M.J. Wright, M.J. Campbell and A. Caracella for obtaining funding for and processing the Australian genotype data. S.M. is supported by an Australian NHMRC Career Development Award.

\section{References}

1. Resnikoff S, et al. Global data on visual impairment in the year 2002. Bull World Health Organ. 2004; 82:844-851. [PubMed: 15640920]

2. Allingham RR, Liu Y, Rhee DJ. The genetics of primary open-angle glaucoma: a review. Exp Eye Res. 2009; 88:837-844. [PubMed: 19061886]

3. Fan BJ, Wang DY, Lam DS, Pang CP. Gene mapping for primary open angle glaucoma. Clin Biochem. 2006; 39:249-258. [PubMed: 16332362]

4. Stone EM, et al. Identification of a gene that causes primary open angle glaucoma. Science. 1997; 275:668-670. [PubMed: 9005853]

5. Rezaie T, et al. Adult-onset primary open-angle glaucoma caused by mutations in optineurin. Science. 2002; 295:1077-1079. [PubMed: 11834836]

6. Monemi S, et al. Identification of a novel adult-onset primary open-angle glaucoma (POAG) gene on 5q22.1. Hum Mol Genet. 2005; 14:725-733. [PubMed: 15677485]

7. Pasutto F, et al. Heterozygous NTF4 mutations impairing neurotrophin-4 signaling in patients with primary open-angle glaucoma. Am J Hum Genet. 2009; 85:447-456. [PubMed: 19765683]

8. Thorleifsson G, et al. Common sequence variants in the LOXL1 gene confer susceptibility to exfoliation glaucoma. Science. 2007; 317:1397-1400. [PubMed: 17690259]

9. Liu Y, et al. Lack of association between LOXL1 variants and primary open-angle glaucoma in three different populations. Invest Ophthalmol Vis Sci. 2008; 49:3465-3468. [PubMed: 18421074]

10. Nakano M, et al. Three susceptible loci associated with primary open-angle glaucoma identified by genome-wide association study in a Japanese population. Proc Natl Acad Sci USA. 2009; 106:12838-12842. [PubMed: 19625618]

11. Rao KN, Kaur I, Chakrabarti S. Lack of association of three primary open-angle glaucomasusceptible loci with primary glaucomas in an Indian population. Proc Natl Acad Sci USA. 2009; 106:E125-126. author reply 106, E127 (2009). [PubMed: 19846772]

12. Jonasson F, et al. Prevalence of open-angle glaucoma in Iceland: Reykjavik Eye Study. Eye (Lond). 2003; 17:747-753. [PubMed: 12928689]

13. Devlin B, Roeder K. Genomic control for association studies. Biometrics. 1999; 55:997-1004. [PubMed: 11315092]

14. Kong A, et al. Detection of sharing by descent, long-range phasing and haplotype imputation. Nat Genet. 2008; 40:1068-1075. [PubMed: 19165921] 
15. Mackey DA, et al. Twins eye study in tasmania (TEST): rationale and methodology to recruit and examine twins. Twin Res Hum Genet. 2009; 12:441-454. [PubMed: 19803772]

16. Tamm ER. The trabecular meshwork outflow pathways: structural and functional aspects. Exp Eye Res. 2009; 88:648-655. [PubMed: 19239914]

17. Gonzalez P, Epstein DL, Borras T. Characterization of gene expression in human trabecular meshwork using single-pass sequencing of 1,060 clones. Invest Ophthalmol Vis Sci. 2000; 41:3678-3693. [PubMed: 11053263]

18. Berta AI, et al. Different caveolin isoforms in the retina of melanoma malignum affected human eye. Mol Vis. 2007; 13:881-886. [PubMed: 17615539]

19. Borrás T. Gene expression in the trabecular meshwork and the influence of intraocular pressure. Prog Retin Eye Res. 2003; 22:435-463. [PubMed: 12742391]

20. Couet J, Belanger MM, Roussel E, Drolet MC. Cell biology of caveolae and caveolin. Adv Drug Deliv Rev. 2001; 49:223-235. [PubMed: 11551396]

21. Hnasko R, Lisanti MP. The biology of caveolae: lessons from caveolin knockout mice and implications for human disease. Mol Interv. 2003; 3:445-464. [PubMed: 14993453]

22. Patel HH, Murray F, Insel PA. Caveolae as organizers of pharmacologically relevant signal transduction molecules. Annu Rev Pharmacol Toxicol. 2008; 48:359-391. [PubMed: 17914930]

23. Parat MO. The biology of caveolae: achievements and perspectives. Int Rev Cell Mol Biol. 2009; 273:117-162. [PubMed: 19215904]

24. Jasmin JF, Yang M, Iacovitti L, Lisanti MP. Genetic ablation of caveolin-1 increases neural stem cell proliferation in the subventricular zone (SVZ) of the adult mouse brain. Cell Cycle. 2009; 8:3978-3983. [PubMed: 19923909]

25. Ju H, Zou R, Venema VJ, Venema RC. Direct interaction of endothelial nitric-oxide synthase and caveolin-1 inhibits synthase activity. J Biol Chem. 1997; 272:18522-18525. [PubMed: 9228013]

26. García-Cardeña G, et al. Dissecting the interaction between nitric oxide synthase (NOS) and caveolin. Functional significance of the nos caveolin binding domain in vivo. J Biol Chem. 1997; 272:25437-25440. [PubMed: 9325253]

27. Fuchshofer R, Tamm ER. Modulation of extracellular matrix turnover in the trabecular meshwork. Exp Eye Res. 2009; 88:683-688. [PubMed: 19385040]

28. Toda N, Nakanishi-Toda M. Nitric oxide: ocular blood flow, glaucoma, and diabetic retinopathy. Prog Retin Eye Res. 2007; 26:205-238. [PubMed: 17337232]

29. Emilsson V, et al. Genetics of gene expression and its effect on disease. Nature. 2008; 452:423428. [PubMed: 18344981]

30. Holm H, et al. Several common variants modulate heart rate, PR interval and QRS duration. Nat Genet. 2010; 42:117-122. [PubMed: 20062063]

31. Frazer KA, et al. A second generation human haplotype map of over 3.1 million SNPs. Nature. 2007; 449:851-861. [PubMed: 17943122] 


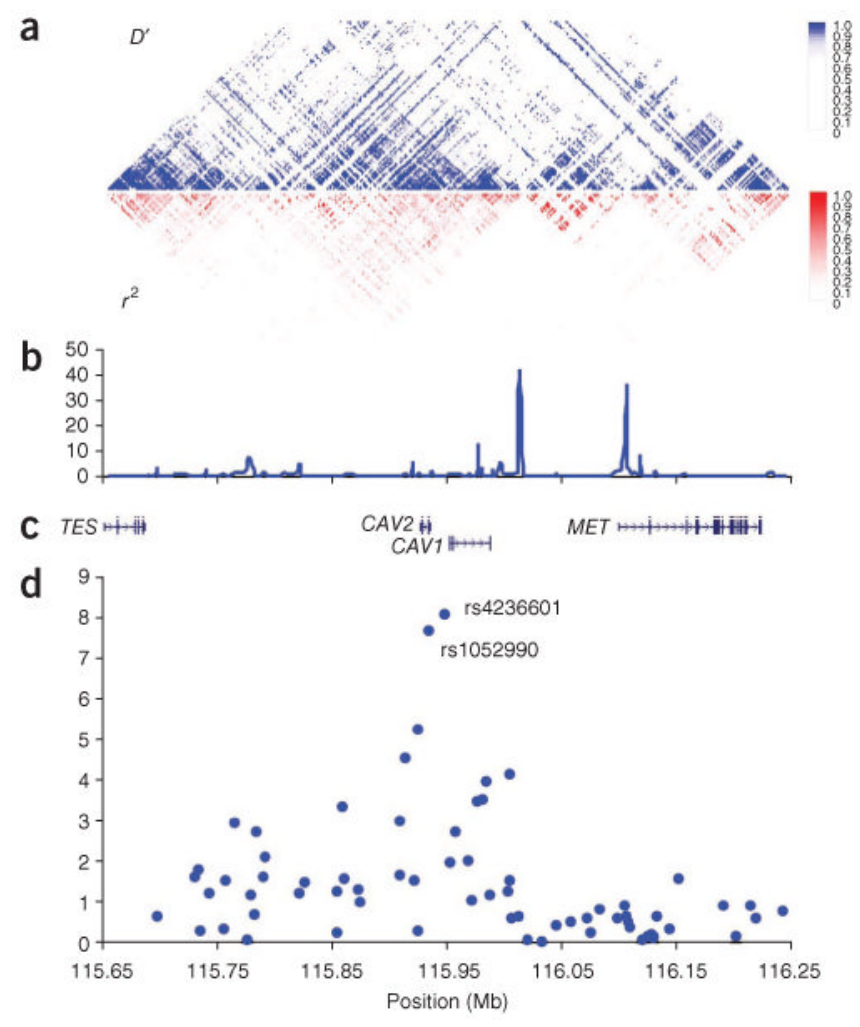

Figure 1.

The 7q31 locus. (a) The pairwise correlation structure in a 600-kb interval (115.65-116.25 $\mathrm{Mb}$, NCBI B36) on chromosome 7. The upper plot shows pairwise $D^{\prime}$ for 533 common SNPs (defined as those having minor allele frequency $>5 \%$ ) from the Utah CEU HapMap (r22) samples. The lower plot shows the corresponding $r^{2}$ values. (b) Estimated sexaveraged recombination rates (saRR) in $\mathrm{cM} / \mathrm{Mb}$ from the HapMap Phase II data ${ }^{31}$. (c) Location of known genes in the region. (d) Schematic view of the association with POAG for all 70 markers tested in the GWAS in the region. All panels use the same horizontal scale shown in $\mathbf{d}$. 


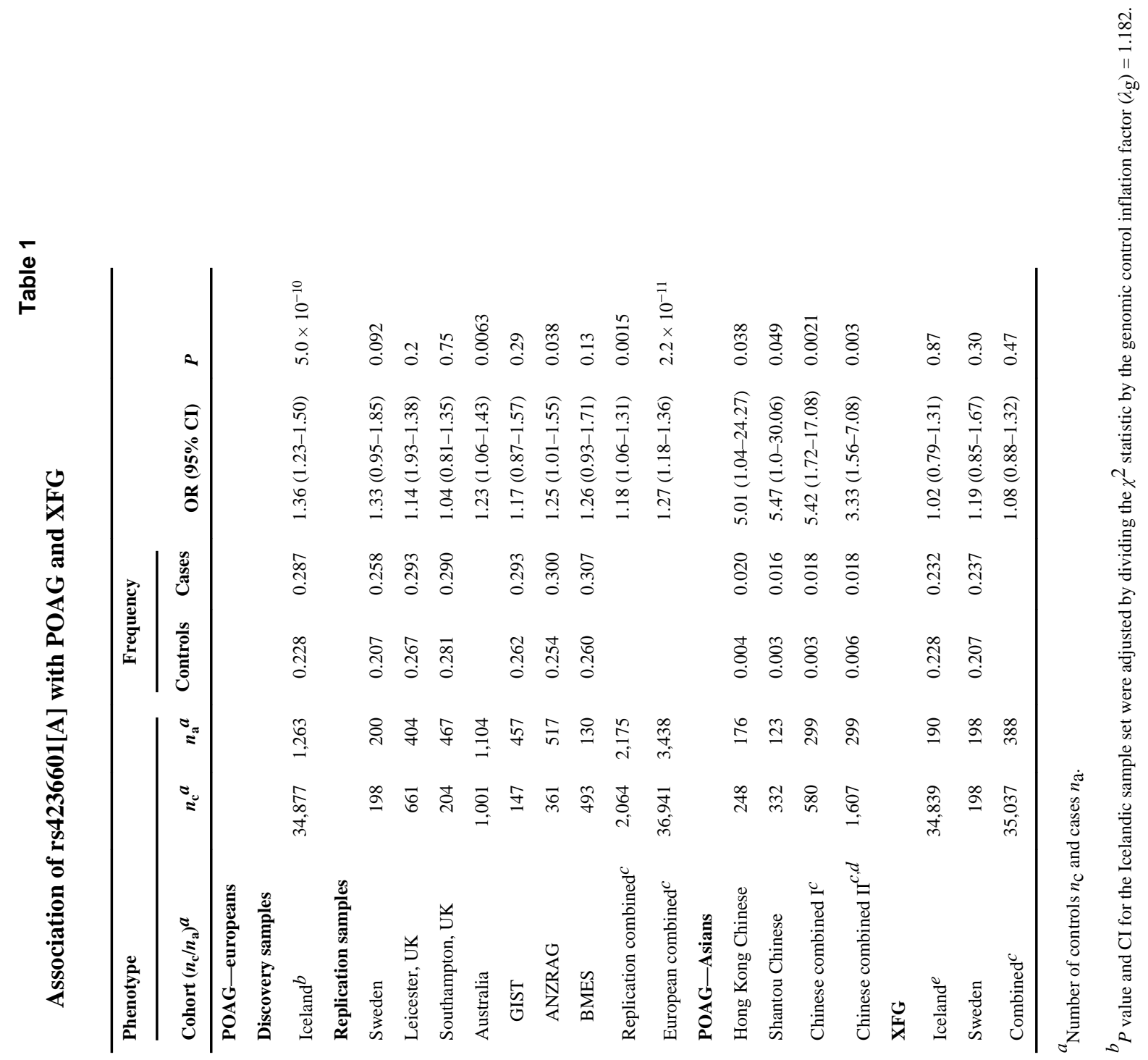




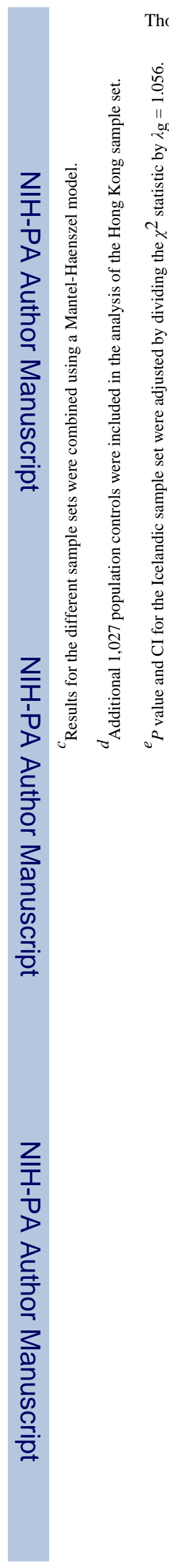

Thorleifsson et al.

Page 10

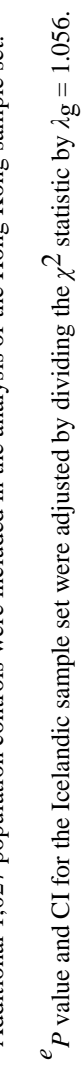

Nat Genet. Author manuscript; available in PMC 2011 November 23. 